\title{
Improving the property profile of a bioresorbable Mg-Y-Nd-Zr alloy by deformation treatments
}

\author{
Natalia Martynenko a,b,*, Elena Lukyanova a,b, Natalia Anisimovab,c, Mikhail Kiselevskiy b,c, \\ Vladimir Serebryany ${ }^{\mathrm{a}}$, Nikita Yurchenko ${ }^{\mathrm{d}}$, Georgy Raab ${ }^{\mathrm{e}}$, Nick Birbilis ${ }^{\mathrm{f}}$, Gennady Salishchev ${ }^{\mathrm{d}}$, \\ Sergey Dobatkin ${ }^{\mathrm{a}, \mathrm{b}}$, Yuri Estrin ${ }^{\mathrm{g}, \mathrm{h}}$ \\ ${ }^{a}$ A.A. Baikov Institute of Metallurgy and Materials Science of the Russian Academy of Sciences, Moscow, Russia \\ ${ }^{\mathrm{b}}$ National University of Science and Technology "MISIS", Moscow, Russia \\ ${ }^{\mathrm{c}}$ N. N. Blokhin National Medical Research Centre of Oncology of the Health Ministry of Russia (N.N. Blokhin NMRCO), Moscow, Russia \\ ${ }^{\mathrm{d}}$ Belgorod National Research University, Belgorod, Russia \\ e Ufa State Aviation Technical University, Ufa, Russia \\ ${ }^{\mathrm{f}}$ College of Engineering and Computer Science, Australian National University, Canberra, Australia \\ ${ }^{\mathrm{g}}$ Department of Materials Science and Engineering, Monash University, Clayton, Australia \\ ${ }^{\mathrm{h}}$ Department of Mechanical Engineering, The University of Western Australia, Crawley, Australia
}

\section{A R T I C L E I N F O}

\section{Keywords:}

Magnesium alloys

Ultrafine grained structure

Mechanical properties

Corrosion resistance

Biocompatibility in vitro

\begin{abstract}
A B S T R A C T
The effect of three deformation methods (equal channel angular pressing (ECAP), multiaxial deformation (MAD), and rotary swaging (RS)) on the structure, texture, mechanical and corrosion properties of magnesium alloy WE43 (Mg-3.56\%Y-2.20\%Nd-0.47\%Zr) was studied. Microstructure, texture, and mechanical properties were investigated by optical and transmission electron microscopy, X-ray diffraction, and tensile testing, respectively. Corrosion resistance was evaluated by a combination of mass loss and hydrogen evolution measurements, as well as electrochemical testing. To assess the effect of the various deformation methods on the biocompatibility in vitro of the alloy, hemolysis and cytotoxicity on peripheral blood cells and proliferation of multipotent mesenchymal stromal cells were evaluated. It tests showed that grain refinement in the range of $\sim 0.6-1 \mu \mathrm{m}$ achieved by mechanical processing is responsible for a significant improvement of mechanical properties. A notable decrease in the corrosion rate was observed after ECAP and MAD processing. MAD and ECAP enhanced alloy biocompatibility in all in vitro tests, while the effect of RS was less significant. In summary, the results obtained demonstrate that not only do the deformation methods employed improve the mechanical properties of alloy WE43, but they also increase its corrosion resistance and biocompatibility in vitro.
\end{abstract}

\section{Introduction}

Presently, orthopedic implants and cardiovascular stents are predominantly fabricated from stainless steel or titanium alloys - both such alloy systems are not biodegradable. To date, such non-biodegradable alloys have worked well in implant applications, but a significant disadvantage is the requirement of a second operation to remove the implants if necessary. Additionally, a non-biodegradable implant placed in a patient's body may also contribute to negative reactions due to the unintended gradual release of metal ions [1]. In addition, the stiffness of stainless steel and titanium alloy implants significantly exceeds that of cortical bone, which can cause uneven bone remodeling due to a stress shielding effect [2,3]. Consequently, significant research efforts are presently focused on developing a new generation of implants that can be similar in their mechanical characteristics to bone tissue, while being bioresorbable, and notionally decomposing in the body during the bone regeneration process. Promising candidates for creating such implants are magnesium-, zinc- and iron-based alloys, as well as degradable polymers. Magnesium and its alloys are potentially favorable bioresorbable materials owing to their biocompatibility and uniquely favorable mechanical properties, which are similar to those of bone tissue [4-9].

However, despite a promising property profile, magnesium-based implant materials have some disadvantages. Firstly, pure magnesium has an excessively fast degradation rate, which leads to a rapid loss of the load-bearing capability of the implant before new bone tissue is formed. Secondly, its biodegradation is accompanied with profuse release of hydrogen gas in the implantation area, which negatively affects the tissues

\footnotetext{
* Corresponding author at: A.A. Baikov Institute of Metallurgy and Materials Science of the Russian Academy of Sciences, Moscow, Russia.

E-mail address: nataliasmartynenko@gmail.com (N. Martynenko).
} 
surrounding the implant and slows down the bone regeneration process $[10,11]$. The corrosion resistance of $\mathrm{Mg}$ can be improved by alloying, which offers a range of possibilities regarding alloy design $[12,13]$. There may be an issue with magnesium alloys, though, as some of them exhibit pitting corrosion $[14,15]$. This can lead to non-uniformity of the mechanical properties of the implant and its premature failure. An interesting avenue to improving the biocorrosion properties of magnesium alloys while at the same time providing the necessary strength and ductility is by modifying their microstructure [16-20]. Therefore, current research into biodegradable implant materials targets magnesium alloys with a microstructure that enables a favourable combination of strength and ductility combined with programmable, uniform corrosion.

A means to moderate the degradation of magnesium is via alloying with specific elements. In addition, consideration to related properties such as strength and ductility, or provide the alloy with antibacterial capability [21] - are also essential. Biocompatibility and the impact of the alloying elements and degradation products on the human body is of paramount importance for the choice of the alloy system. At present, zinc [22,23], calcium [24,25], silver [26,27], manganese [28,29], strontium [30], lithium [31,32], some rare-earth metals (yttrium, neodymium [33,34], gadolinium [27, 35]) and, in small quantities, aluminum [33, 36,37] (despite its potentially harmful effect on the human body [38]) are the most common alloying elements studied in the context of Mg-based alloys. Zirconium (Zr) is also present in some alloys as a structural modifier (grain refiner). The addition of these elements (rare-earth metals) to magnesium has a positive effect on the corrosion resistance in biomedical environments and enhance Mg-alloy strength.

In order to improve the mechanical properties, deformation processing can be effectively applied to Mg-alloys as there is potential to strengthen Mg-alloys from grain refinement owing to the Hall-Petch coefficient for Mg-alloys, with grain refinement not necessarily having a negative impact on alloy ductility (although, this may also be dependent on the specific crystallographic textures imparted). It is necessary to find such methods of deformation processing that would bring about an improvement of mechanical properties without sacrificing the corrosion resistance. For these purposes, the formation of an ultrafine-grained (UFG) structure in magnesium alloys can be appropriate. Not only does the formation of an UFG, i.e. submicron scale, grain structure lead to a significant strengthening of magnesium alloys, but it does not impair their corrosion resistance, and in some cases even improves it [39]. In addition, the formation of a UFG structure often helps to solve a further problem with magnesium alloys, viz. the occurrence of pitting corrosion, i.e. localisation of corrosion processes leading to undesired nonuniformity of the mechanical properties of the implant [40]. Therefore, obtaining magnesium alloys with a deformation-induced UFG structure is a promising avenue to improved implant materials.

Severe plastic deformation (SPD) is an established method for imparting a UFG structure to metallic materials [40]. Among the SPD techniques, high pressure torsion (HPT) and equal-channel angular pressing (ECAP) are the most popular ones [41]. The first method is a good way to simulate the processes occurring in a metal under the most severe conditions of deformation, while the second allows getting massive workpieces with UFG structure [41]. In addition, in recent years, many research works aimed at studying the possibility of obtaining UFG structures in metals and alloys by traditional metal working techniques, which, due to their relatively low cost and greater commercial viability, are sometimes preferable to SPD methods. Traditional techniques include rotary swaging (RS) [42-44], multiaxial deformation (MAD) often referred to as isothermal forging $[45,46]$, and radial shear rolling (RSR) [47]. These techniques enable refinement of the structure and the ensuing substantial strengthening of metallic materials, including magnesium and its alloys, in a very efficient way. Besides, these processing steps can easily be integrated in the current production cycles at industrial scale. These considerations motivated the present study of the effect of two conventional metal working techniques, alongside the SPD treatment by equal-channel angular pressing, on the structure and the associated mechanical and in-service properties of one of the most popular bioresorbable Mg-based implant materials: alloy WE43. While some of the pertinent properties of the alloy were reported in literature [33,34, 48-52], its full property profile relevant for applications in medical implants is not established so far. Below we present a comprehensive account of the effect of MAD, RS, and ECAP on microstructure, texture, mechanical and corrosion properties, and in-vitro biocompatibility of alloy WE43.

\section{Experimental}

The present study explored magnesium alloy WE43, which was determined according to chemical analysis of cast ingots contained $3.56 \%$ Y, 2.20\% Nd and $0.47 \% \mathrm{Zr}$ (in wt. \%). The cast alloy was homogenized at $525^{\circ} \mathrm{C}$ for 8 hours, and ingots were then extruded at $430^{\circ} \mathrm{C}$ to obtain rods with a diameter necessary for subsequent deformation processing (25 mm - for MAD, $20 \mathrm{~mm}$ - for RS and $10 \mathrm{~mm}$ - for ECAP). The extrusion ratio ${ }^{1}$ at this pre-processing step was 28.4 for deformation by equal channel angular pressing, 10.2 by rotary swaging and 6.6 by multiaxial deformation. Upon deformation processing, the rods were annealed again at $525^{\circ} \mathrm{C}$ for 8 hours and air cooled to create a supersaturated solid solution of rare-earth metals in magnesium. In the following, the condition of the alloy produced by this chain of processing steps will be referred to as the initial state.

The processing steps for the various deformation methods and processing schedules employed in this study are presented in Fig. 1. Multipass Route Bc ECAP (with billet rotation by $90^{\circ}$ between the passes) was conducted using a die with a $120^{\circ}$ angle between the entry and exit channels, with a stepwise decrease of temperature from the initial $425^{\circ} \mathrm{C}$ to $300^{\circ} \mathrm{C}$ at the final, $12^{\text {th }}$ pass. The cumulative equivalent strain the ECAP billets underwent was about 7.8. Multiaxial deformation with a stepwise decrease of temperature from $450{ }^{\circ} \mathrm{C}$ at the first cycle to 300 ${ }^{\circ} \mathrm{C}$ at the final, 28 th cycle was also carried out. The cumulative equivalent strain imparted on the MAD billets was about 17.5. The experiments were conducted on an Instron 300LX universal hydraulic testing machine at a deformation speed of $2 \mathrm{~mm} / \mathrm{min}$. Rotary swaging was carried out on an RKM 2129.02 rotary swaging machine (with a maximum force of $8 \mathrm{kN}$ ) with a decrease in temperature from 400 to $325{ }^{\circ} \mathrm{C}$. The machine had the frequency of $1920 \mathrm{~min}^{-1}$ and the striker stroke of 3 $\mathrm{mm}$. Accordingly, there were eight hits of strikers for one revolution of the billet about its axis. The billets were deformed in two stages, first at $400^{\circ} \mathrm{C}$ and then at $325^{\circ} \mathrm{C}$, to a cumulative strain to $\varepsilon=0.94^{2}$.

The microstructure of the alloy in the initial state was studied by optical microscopy using an Axio Observer D1m Carl Zeiss light microscope. The microstructure formed after treatment by various deformation methods was studied by transmission electron microscopy (TEM) using a JEM-1400 electron microscope (JEOL, Japan) operating at a voltage of $120 \mathrm{kV}$. Specimen foils were first mechanically thinned to $180 \mu \mathrm{m}$ and then subjected to ion bombarded in a GATAN 600 unit. The size of the structural elements was measured by random intercept method using Image Expert Professional 3 software.

The texture formed in the billets in the longitudinal direction was examined using a DRON-7 X-ray texture diffractometer in $\mathrm{CuK}_{\alpha}$ radiation in the reflection mode by recording five incomplete $\{00.4\}$, $\{21.1\},\{10.2\},\{10.3\}$, and $\{11.0\}$ pole figures, at a maximum tilt angle $\alpha_{\max }=70^{\circ}$ and at a step of $5^{\circ}$ for $\alpha$ and $\beta\left(0-360^{\circ}\right.$ ) (where $\alpha$ and $\beta$ are the radial and azimuth angles in a pole figure, respectively). The orientation distribution functions (ODFs) and the volume fractions of the main orientations were calculated using approximation by a large number of Gaussian normal distributions [53]. Quantitative analysis of textures by this method makes it possible to evaluate the generalized

\footnotetext{
1 The extrusion ratio was calculated using the formula: $\mu=\mathrm{A}_{0} / \mathrm{A}_{\mathrm{f}}$, where $\mathrm{A}_{0}$ and $A_{f}$ are the initial and the final cross-section areas of the billets, respectively. ${ }^{2} \varepsilon=\ln \left(\mathrm{A}_{0} / \mathrm{A}_{\mathrm{f}}\right)$, where $\mathrm{A}_{0}$ and $\mathrm{A}_{\mathrm{f}}$ are the initial and the final cross-section areas of the billets, respectively.
} 


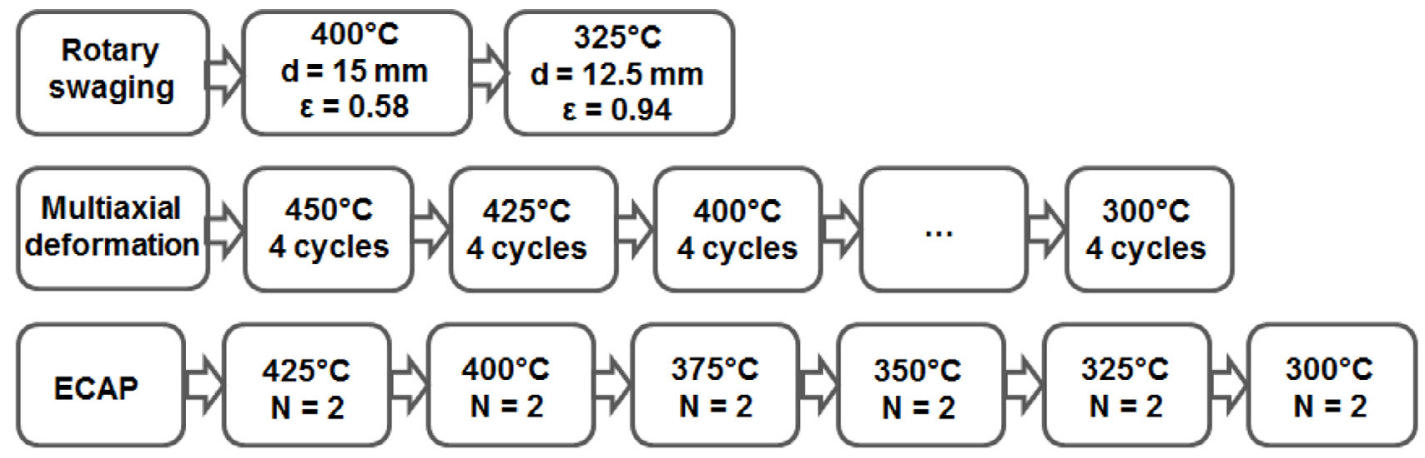

(a)

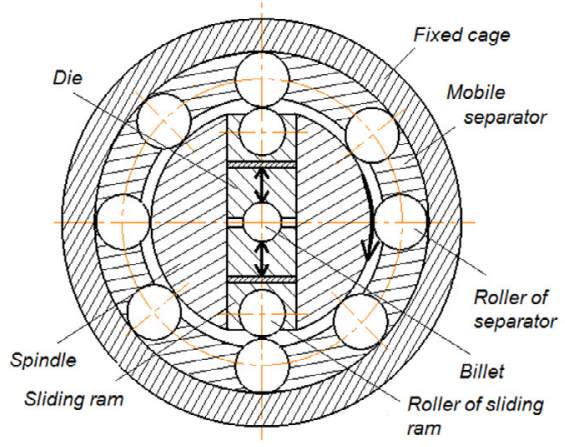

(b)

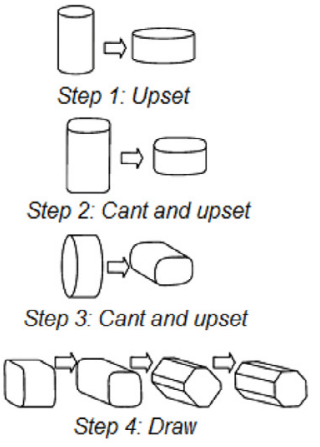

(c)

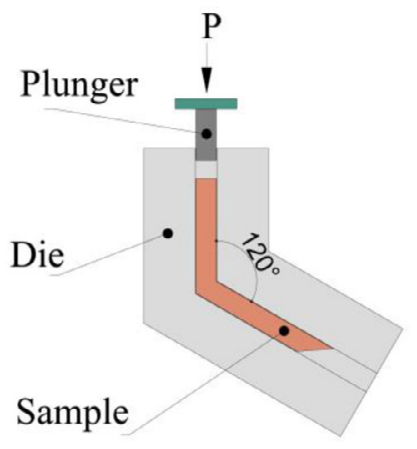

(d)

Fig. 1. The processing histories of the alloy (a) and the schematics of RS (b), MAD (c) and ECAP (d) processes.

Schmid factors for active deformation systems in the studied material. The orientation factors depend on the generalized Schmid factors according to the following relations [54]:

$M_{i}=\frac{1}{m_{i}}$

where $m_{i}$ denotes the generalized Schmid factors for basal and prismatic slip and twinning. They can be estimated for a selected deformation system and the texture of the alloy using the following relation:

$m_{i}=\sum_{j=1}^{p} m_{i j} W_{j}$

where $m_{i j}$ is the orientation Schmid factor calculated for the ith slip system and the $j$ th texture component, $\mathrm{W}_{\mathrm{j}}$ is the volume fraction of the $j$ th texture component, and $p$ is the number of texture components.

The mechanical properties of alloy WE43 with the various processing histories were determined by uniaxial tensile tests on an Instron 3382 testing machine with a strain rate of $1 \mathrm{~mm} / \mathrm{min}$ at room temperature. Cylindrical specimens with the diameter of $3 \mathrm{~mm}$, the total length of 33 $\mathrm{mm}$, and the gage length of $15 \mathrm{~mm}$ were used.

The corrosion resistance of the alloy was evaluated based on mass loss, hydrogen evolution, and the potentiodynamic polarization (PDP) measurements. A physiological saline solution $(0.9 \% \mathrm{NaCl}$ dissolved in distilled water, $\mathrm{pH}=7$ ) was used as a test medium. The samples for the tests were prepared by manual mechanical grinding on abrasive paper (from P800 to P2500). The mass loss and the hydrogen evolution tests were carried out at $37^{\circ} \mathrm{C}$, while PDP tests were conducted at room temperature. After immersion in a corrosive environment and removal of the corrosion products by cleaning in a mixture of $\mathrm{Cr}_{2} \mathrm{O}_{3}, \mathrm{AgNO}_{3}$, $\mathrm{Ba}\left(\mathrm{NO}_{3}\right)_{2}$ and reagent water for 1 min (ASTM_G1-03-E), a specimen was weighed to determine the corrosion rate by the mass loss method. The mass change was determined by weighing on an electronic scale GR 200 with an accuracy of the fourth digit. Electrochemical tests were carried out on a VMP3 potentiostat, under the control of EC-Lab software (BioLogic, France). The experimental setup included a PAR flat cell with a "three electrode configurations" (a working electrode, a saturated calomel reference electrode, and a Pt-mesh counter electrode). Scanning was carried out at a speed of $1 \mathrm{mV} / \mathrm{s}$ in a range from $100 \mathrm{mV}$ below the open circuit potential to $-1000 \mathrm{mV}$. The exposure time before the start of the scan, necessary for the surface to reach a stable potential (determination of the open circuit potential), was 10 minutes. For each test sample five scans were conducted.

Biocompatibility in vitro was determined by assessing hemolysis in red blood cells (RBC) suspension, viability of white blood cells (WBC), and proliferation of multipotent mesenchymal stromal cells (MMSC). Measurements of the biodegradation rate of alloy were carried out in fetal bovine serum (FBS, HyClone UK Ltd., Thermo Scientific). Pennyshaped samples used in biocompatibility ( 9 pieces of each states) and biodegradation (3 pieces of each states) assays had a diameter of 8 $\mathrm{mm}$ and a thickness of $2 \mathrm{~mm}$. They were ground on abrasive paper (P2000) and then polished on wet cloth. Before the tests, the samples were washed with distilled water, sterilized by autoclaving at a temperature of $120^{\circ} \mathrm{C}$ for 30 minutes under a pressure of $1 \mathrm{~atm}$, and dried in a sterile atmosphere. The study of hemolysis and cell viability was carried out using WBCs and RBCs of CBA mice (with a mass of $22-24 \mathrm{~g}$ ) in accordance with the methods described previously [55]. In brief, each sample was incubated in $1 \mathrm{ml}$ of a suspension of RBCs and WBC for $24 \mathrm{~h}$ at $37^{\circ} \mathrm{C}$ in an atmosphere of $5 \% \mathrm{CO}_{2}$. Intact cells incubated under the same conditions were used as a control. To evaluate hemolysis, the optical density (OD) of the supernatant was measured in triplets after 1, 2, 3, 4, 5 and 24 hours of incubation with alloy samples using an the MS Multiscan (Thermo Fisher) plate reader at $540 \mathrm{~nm}$. The cells viability assessment after incubation with alloy samples and in the control was performed using the MTT test after 2 and $24 \mathrm{~h}$ of incubation by measuring the OD in triplets on the plate reader at $540 \mathrm{~nm}$. MMSCs were generated according 
to standard methods, isolating progenitor cells from the bone marrow of CBA mice [56]. The study of the effect of samples on the proliferation of MMSCs was carried out in the way described [57]. The main steps were as follows: three samples of each alloy were preincubated overnight in RPMI-1640 medium (Sigma-Aldrich); 540,000 cells were seeded onto the alloy surface in 24-well plates with agarose-coated bottom, allowed to adhere for 30 minutes, and then $2 \mathrm{ml}$ RPMI-1640 medium (SigmaAldrich) supplemented with $10 \%$ FBS, 4mM L-glutamine and 1\% penicillin/streptomycin (both PanEco, Russia) was added to each well. Cells were further cultured for 7 days at $37^{\circ} \mathrm{C}$ for adhesion in an atmosphere with $5 \% \mathrm{CO}_{2}$. The growth medium was changed daily. Cells treated with the growth medium only were used as a control. The cell proliferation in fresh growth medium was investigated in an MTT assay. OD of the samples was measured in triplets on the plate reader at $540 \mathrm{~nm}$. The difference between the counts for the samples of the processed alloy and that in the initial state was calculated in percent ( $\%$ of Initial state) to assess the impact of the deformation methods on the biocompatibility parameters of the WE43 alloy. Statistical analysis of the obtained data was performed by calculating the mean value and the standard deviation. Comparative analysis was performed in the t-test. The difference between the value for a processed alloy and that for the initial alloy was considered statistically significant at $\mathrm{p}<0.05$.

The degradation rate of alloy WE43 was estimated by incubating each sample in $2 \mathrm{ml}$ of FBS at $37^{\circ} \mathrm{C}$ in an atmosphere of $5 \% \mathrm{CO}_{2}$ for $1,2,3$, and 4 weeks. It was quantified by the relative mass loss $\left(m_{o^{-}}\right.$ $\left.m_{f}\right) / m_{0} \times 100 \%$, where $m_{f}$ and $m_{o}$ denote the final and the initial mass of the sample. The sample surfaces after degradation tests were investigated using an MMI-2 instrumental microscope.

The parameters of the roughness of the sample surface were measured to evaluate its effect on the corrosion properties and biocompatibility of the alloy. Roughness measurements were carried out using a WYKO NT 1100 optical profilometer (Veeco Instruments, USA) using vertical scanning interferometry. The average roughness was measured over a surface area of $900 \times 1,200 \mu \mathrm{m}$ for each microstructural state of the alloy.

The cell and animal test protocols used in this work were evaluated and approved by the local Ethics Committee of N.N. Blokhin National Medical Research Centre of Oncology" of the Health Ministry of Russia.

\section{Results}

Fig. 2 displays the microstructure of WE43 alloy in the initial state and after the deformation treatments employed in this study. Optical microscopy image in Fig. 2a corresponding to the initial state exhibits a uniform structure consisting of equiaxed grains of a magnesium solid solution with an average grain size $d$ of about $65 \mu \mathrm{m}$. The averaging was done over microstructures in three 'initial states' corresponding to pre-processing for subsequent ECAP, MAD, and RS. As mentioned in Section 2, this pre-processing involved different diameter reductions for the three deformation modes considered. We note, however, that after annealing, the individual values of the average grain size for these three extrusion histories did not differ within the measurement error. A further microstructural feature of note is the occurrence of small inclusions of the $\mathrm{Mg}_{41} \mathrm{Nd}_{5}$ phase at grain boundaries [52, 58], which did not dissolve during annealing (Fig. 2a).

All three deformation methods led to the formation of an UFG structure (Fig. 2 b-e). After multiaxial deformation, the average grain size was $0.93 \pm 0.29 \mu \mathrm{m}$. ECAP led to a refinement of the initial grain structure down to $0.69 \pm 0.13 \mu \mathrm{m}$, while rotary swaging reduced the average grain size to $0.61 \pm 0.19 \mu \mathrm{m}$. In the latter case, the formation of twins with a width of $0.4-0.8 \mu \mathrm{m}$ was also observed. It should also be noted that the presence of point reflexes on the electron diffraction ring pattern indicates that the microstructure formed by the deformation processing had predominantly higher-angle grain misorientations. In addition, the combination of pre-heating for processing and the deformation process itself also led to precipitation of dispersed particles of
Table 1

The main orientations (hkil)[uvtw] and their volume fractions $\left(\mathrm{W}_{\mathrm{i}}\right)$ for various microstructural states of alloy WE43.

\begin{tabular}{|c|c|c|c|}
\hline Treatment & (hkil) & [uvtw] & $\mathrm{W}_{\mathrm{i}}, \%$ \\
\hline \multirow{6}{*}{ Initial state for ECAP } & $(0001)$ & {$[\overline{1} \overline{5} 60]$} & 2 \\
\hline & $(\overline{1} 2 \overline{1} .23)$ & {$[13 . \overline{8} \overline{5} 1]$} & 2 \\
\hline & $(\overline{1} 2 \overline{1} .14)$ & {$[\overline{8} \overline{5} .13 .1]$} & 2 \\
\hline & $(\overline{2} 4 \overline{2} .11)$ & {$[\overline{4} \overline{2} 61]$} & 2 \\
\hline & $(\overline{1} 2 \overline{1} 3)$ & {$[3 \overline{1} \overline{2} 1]$} & 2 \\
\hline & non-textured material & & 90 \\
\hline \multirow{6}{*}{ ECAP } & $(\overline{1} 2 \overline{1} 0)$ & [10̄11] & 4 \\
\hline & $(\overline{1} 2 \overline{1} 0)$ & {$[10 \overline{1} 2]$} & 5 \\
\hline & $(\overline{1} 2 \overline{1} 0)$ & [1]018] & 5 \\
\hline & $(\overline{1} 2 \overline{1} 0)$ & {$[\overline{3} 034]$} & 4 \\
\hline & $(\overline{1} 2 \overline{1} 0)$ & [2027] & 3 \\
\hline & non-textured material & & 79 \\
\hline \multirow[t]{6}{*}{ Initial state for MAD } & $(\overline{1} 2 \overline{1} 0)$ & {$[10 \overline{1} 0]$} & 1 \\
\hline & $(\overline{1} 2 \overline{1} 4)$ & {$[\overline{1} \overline{6} 75]$} & 1 \\
\hline & $(\overline{2} 6 \overline{4} 5)$ & [15̄59] & 1 \\
\hline & $(25 \overline{7} .33)$ & {$[2 \overline{7} 52]$} & 1 \\
\hline & $(59 \overline{14} 2)$ & [1]019] & 1 \\
\hline & non-textured material & & 95 \\
\hline \multirow[t]{6}{*}{ MAD } & $(12 \overline{3} 3)$ & {$[1 \overline{11} .10 .17]$} & 1 \\
\hline & $(39 \overline{12} 1)$ & {$[4 \overline{3} \overline{1} 3]$} & 0.6 \\
\hline & $(02 \overline{2} 5)$ & {$[10 . \overline{11} 15]$} & 0.6 \\
\hline & $(\overline{1} 4 \overline{3} 1)$ & {$[10 . \overline{3} \overline{7} 2]$} & 0.6 \\
\hline & $(\overline{1} 4 \overline{3} 8)$ & {$[3 \overline{3} 02]$} & 0.6 \\
\hline & non-textured material & & 96.6 \\
\hline
\end{tabular}

Table 2

The mechanical properties of alloy WE43 in the initial state (average values for three «initial states») and after different deformation treatments.

\begin{tabular}{lllll}
\hline Microstructural state & YS, MPa & UTS, MPa & El, $\%$ & $d, \mu \mathrm{m}$ \\
\hline Initial state & 161 & 234 & 9 & $\sim 65$ \\
ECAP & 260 & 300 & 13.2 & $0.69 \pm 0.13$ \\
MAD & 210 & 300 & 17.2 & $0.93 \pm 0.29$ \\
RS $\left(325{ }^{\circ} \mathrm{C}, \mu=2.56\right)$ & 287 & 416 & 7.9 & $0.61 \pm 0.19$ \\
\hline
\end{tabular}

the $\mathrm{Mg}_{41} \mathrm{Nd}_{5}$ phase. The average particle size was $0.34 \pm 0.21 \mu \mathrm{m}, 0.45$ $\pm 0.18 \mu \mathrm{m}$, and $0.31 \pm 0.09 \mu \mathrm{m}$ for MAD, ECAP, and RS, respectively.

The results of the texture analysis of the alloy in the respective initial state and after deformation by MAD and ECAP are presented in Fig. 3 and Table 1.

As can be seen from Fig. 3, the strain produced by extrusion leading up to what we call the initial state had a strong effect on the texture of the alloy. A weak basal texture inclined to the center of the pole figure $(00.4)$ by $50-60^{\circ}$ was formed in the alloy after extrusion with an extrusion ratio of 6.6 and subsequent annealing, i.e. the pre-processing for MAD (Fig. 3a). Calculations showed the presence of five basal orientations, the volume fraction of which did not exceed $1 \%$, and the share of the non-textured component was $95 \%$ (Table 1). By contrast, a sharp basal texture was observed after extrusion with an extrusion ratio of 28.4 and subsequent annealing, i.e. the pre-processing for ECAP (Fig. 3c). The texture formed encompassed five basal orientations, each with a volume fraction of $2 \%$. The share of the non-textured component in this case is $90 \%$ (Table 1 ).

The two modes of severe plastic deformation used in subsequent processing also had different effects on the texture of the studied alloy. Thus, after MAD, the main orientations changed, and the initial texture was much more scattered (Fig. 3b). In this case, the texture is also characterized by five different 1 orientations whose total volume fraction does not exceed $3.4 \%$, and the share of the non-textured component grows to $96.6 \%$. Not only an increase in the sharpness of the texture, but also a transformation from a basal texture to a prismatic one was discovered in the case of ECAP (Fig. 3d). The ECAP-induced texture is characterized by five basal orientations, the share of the non-textured component being reduced to $79 \%$. 


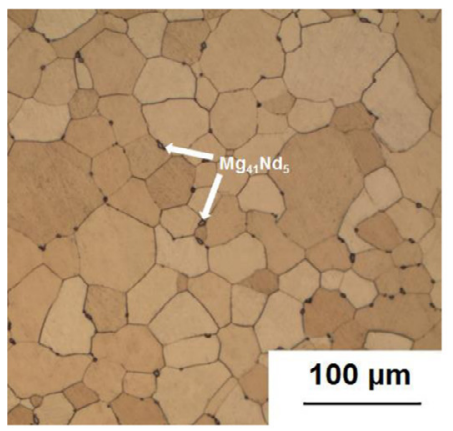

(a)

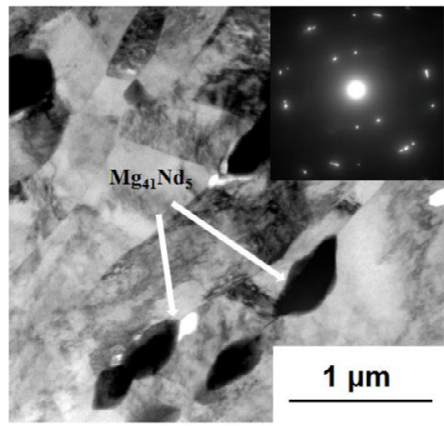

(d)

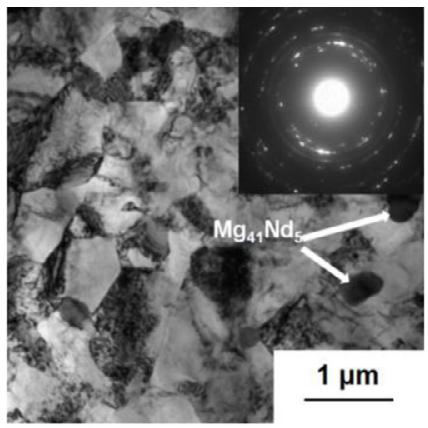

(b)

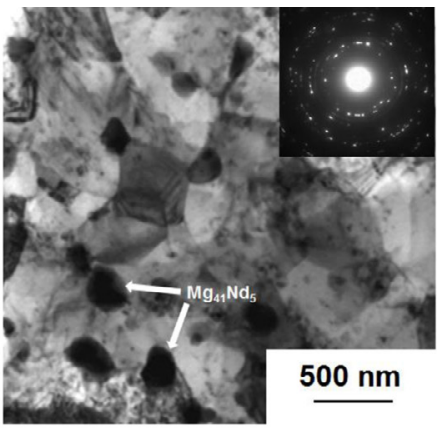

(c)

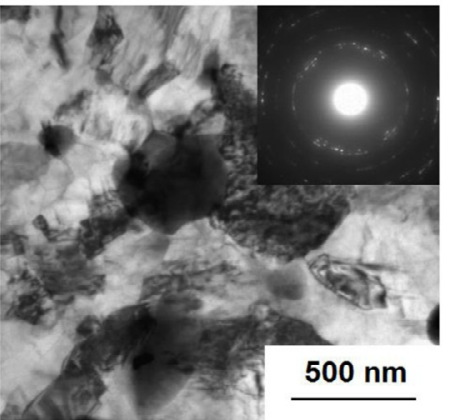

(e)

Fig. 2. The structure of the alloy for the initial state (a), as well as after ECAP (b), MAD (c), and RS at $325^{\circ} \mathrm{C}(\varepsilon=0.94)(d, e)$.
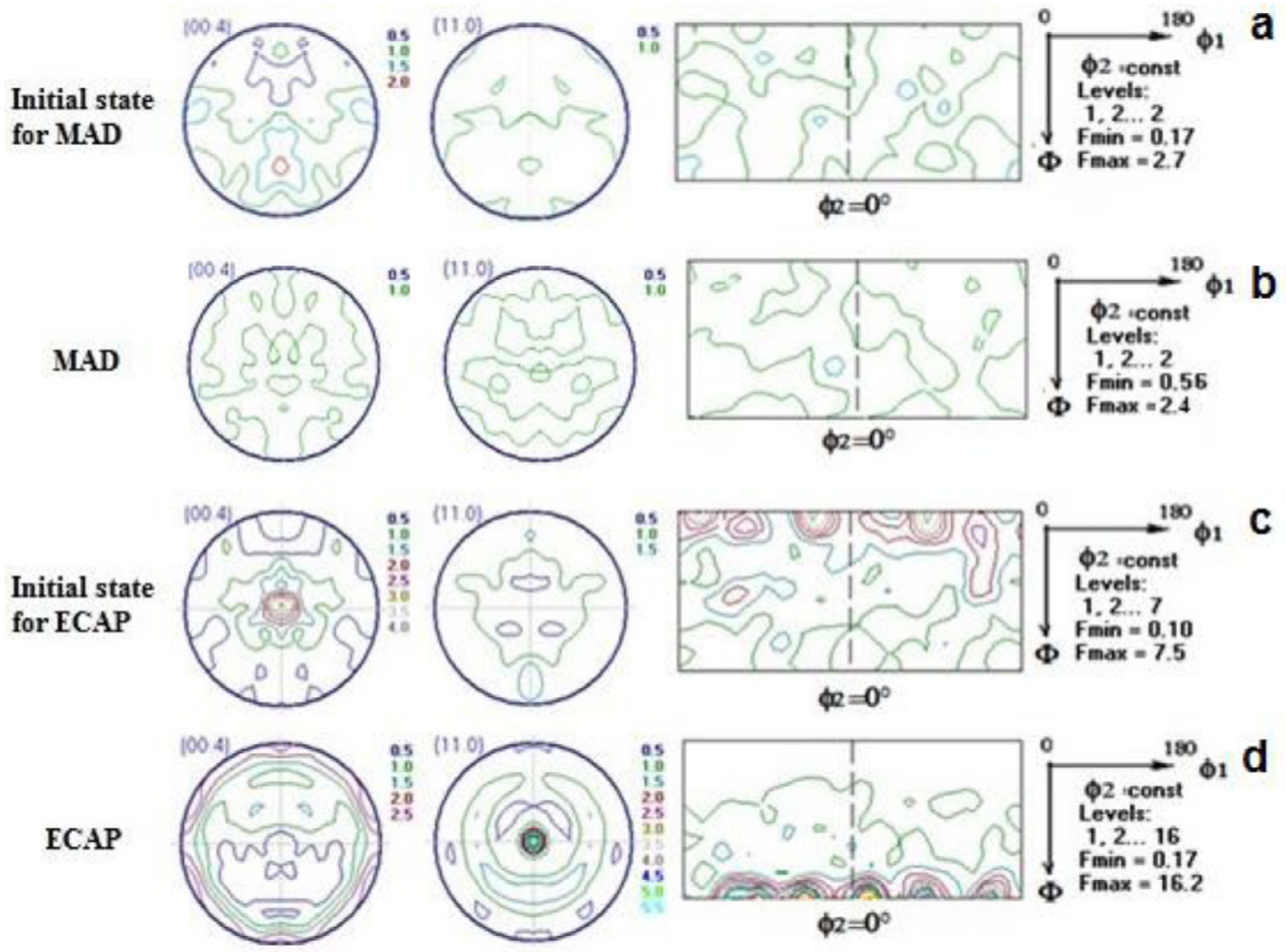

Fig. 3. The texture of the alloy in the initial state before deformation (a, c) and after processing by MAD (b) and ECAP (d). 


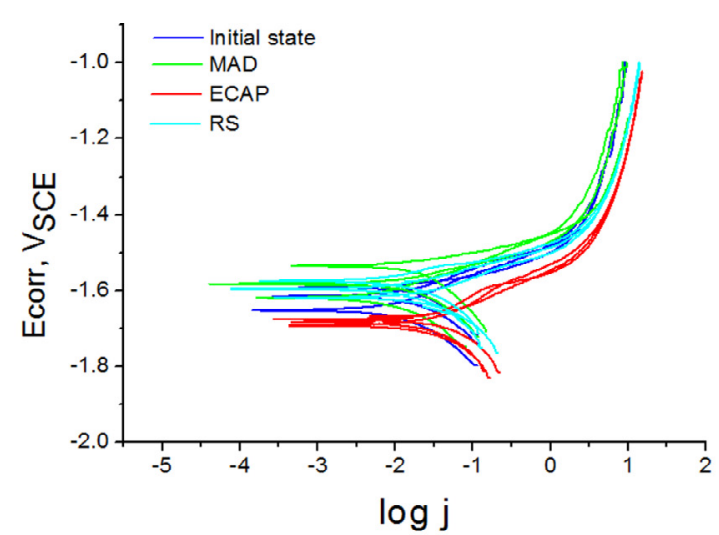

(a)

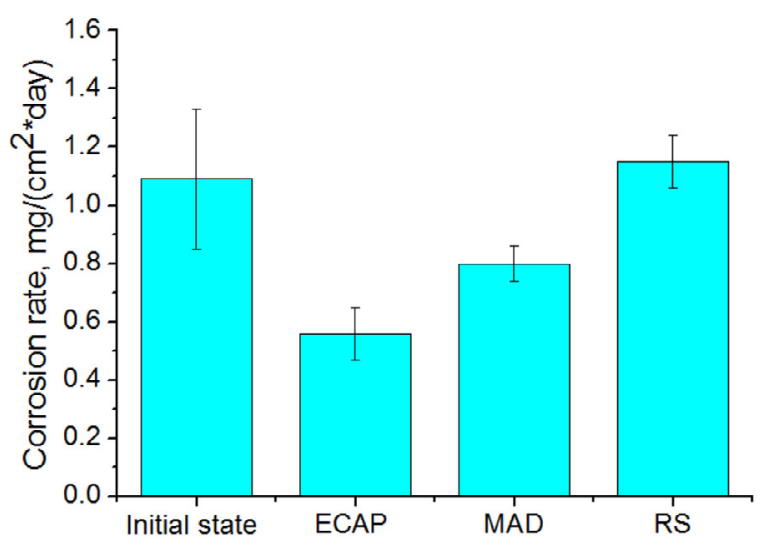

(c)

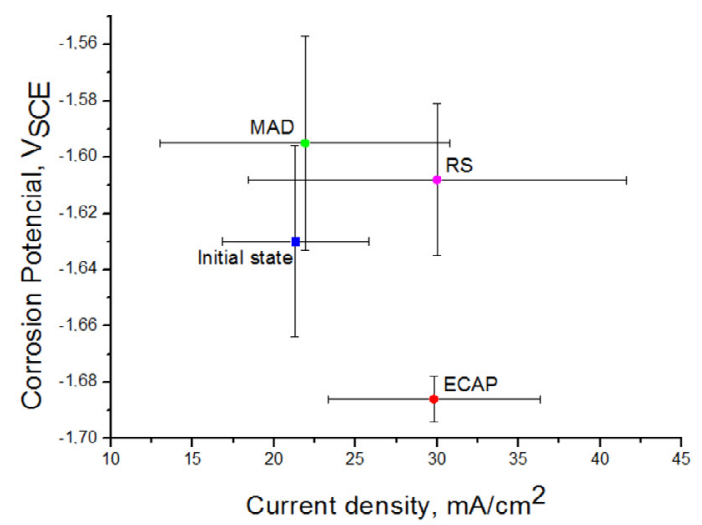

(b)

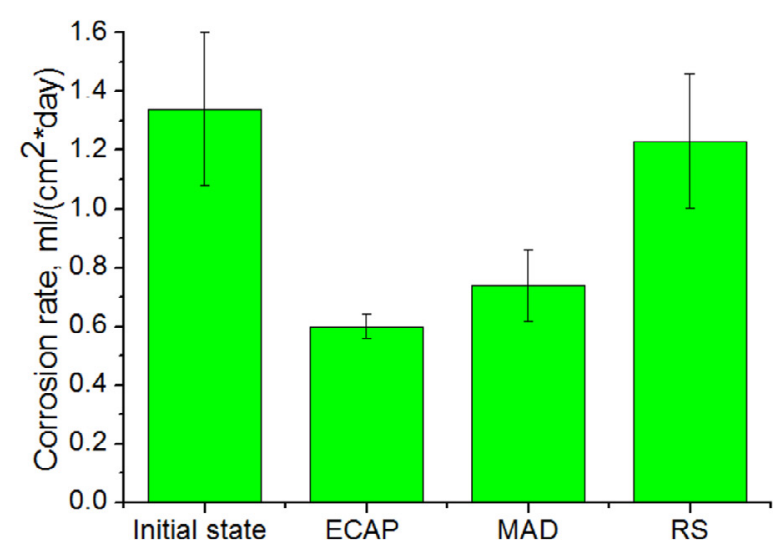

(d)

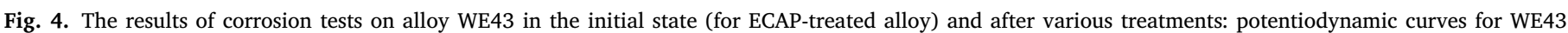

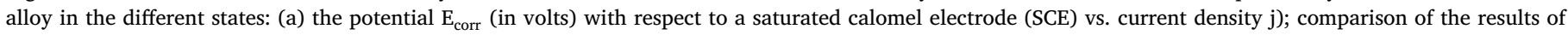
potentiodynamic tests (b); mass loss (c) and hydrogen evolution (d).

Table 2 summarizes the mechanical properties of alloy WE43 in the initial state and after the deformation treatments investigated. It is seen that the formation of a UFG structure in the process of deformation leads to strengthening of the WE43 alloy. Thus, an increase in the yield stress (YS) of the alloy from $161 \mathrm{MPa}$ in the initial state to $260 \mathrm{MPa}$ after ECAP processing was observed. The ultimate tensile strength (UTS) in this case rose from 234 to $300 \mathrm{MPa}$. In the case of MAD, the yield stress after processing reached $210 \mathrm{MPa}$ and the ultimate tensile strength was $300 \mathrm{MPa}$. However, grain refinement was most pronounced for rotary swaging, which gave rise to the greatest increase in the strength characteristics, the yield stress and the ultimate tensile strength reaching the values of 287 and $416 \mathrm{MPa}$, respectively. The tensile elongation (El) of the WE43 alloy increased from 9\% in the initial state to 13.2 and $17.2 \%$ after ECAP and MAD, respectively, along with an increase in strength. In the case of rotary swaging, a slight drop in tensile elongation from 9 to $7.9 \%$ was observed. It should also be noted that extrusion prior to annealing did not have any significant effect on the mechanical properties of the alloy in the initial state.

Fig. 4 shows the corrosion resistance results for WE43 in the initial state and after deformation processing based on measurements by three different methods: potentiodynamic polarization at room temperature, mass loss, and hydrogen evolution measurements. All tests were performed in physiological saline solution $(0.9 \% \mathrm{NaCl}$ dissolved in distilled water).

The investigation of the corrosion resistance using the PDP tests showed that the structure formed after all the studied types of deforma- tion did not affect the resistance of alloy WE43 to electrochemical corrosion. The magnitude of the corrosion potential of the alloy remained the same within the error range for all four states and were equal to $-1.630 \pm 34 \mathrm{mV}$ for the initial state of the alloy, $-1686 \pm 8 \mathrm{mV}$ for the alloy after ECAP, $-1595 \pm 38 \mathrm{mV}$ after MAD, and $-1608 \pm 27 \mathrm{mV}$ after RS. The corrosion current density values were about the same within the measurement error: $21.3 \pm 4.5 \mu \mathrm{A} / \mathrm{cm}^{2}, 21.8 \pm 6.5 \mu \mathrm{A} / \mathrm{cm}^{2}, 21.9$ $\pm 89 \mu \mathrm{A} / \mathrm{cm}^{2}$, and $30.0 \pm 11.6 \mu \mathrm{A} / \mathrm{cm}^{2}$ for the alloy in the initial state and after ECAP, MAD, and RS, respectively.

As distinct from the PDP results, the data on the resistance to chemical corrosion showed a slightly different tendency. The grain refinement by ECAP and MAD slowed down the chemical corrosion at $37^{\circ} \mathrm{C}$. In the initial, coarse-grained state, the mass loss of the alloy was $1.09 \pm 0.24$ $\mathrm{mg} / \mathrm{cm}^{2} *$ day, whereas after ECAP and MD this quantity amounted to $0.56 \pm 0.09 \mathrm{mg} / \mathrm{cm}^{2} *$ day and $0.80 \pm 0.06 \mathrm{mg} / \mathrm{cm}^{2} *$ day, respectively. An exception was the case of RS, when the structure formed by the process did not affect the chemical corrosion rate $\left(1.09 \pm 0.24 \mathrm{mg} / \mathrm{cm}^{2} *\right.$ day $v s .1 .15 \pm 0.09 \mathrm{mg} / \mathrm{cm}^{2} *$ day for the initial state). Hydrogen evolution exhibited a similar tendency: ECAP and MD were found to reduce its rate, while RS did not produce any sizeable effect. The corrosion rates calculated from the hydrogen evolution data were as follows: $1.34 \pm 0.26$, $0.60 \pm 0.04,0.74 \pm 0.12$, and $1.23 \pm 0.23 \mathrm{ml} / \mathrm{cm}^{2} *$ day for the initial state of the alloy and upon ECAP, MAD, and RS, respectively.

The effect of deformation treatment on the biocompatibility of WE43 in the initial state was studied in vitro by evaluating hemolysis (destruction of RBCs), WBCs viability, and MMSC proliferation after incubation 


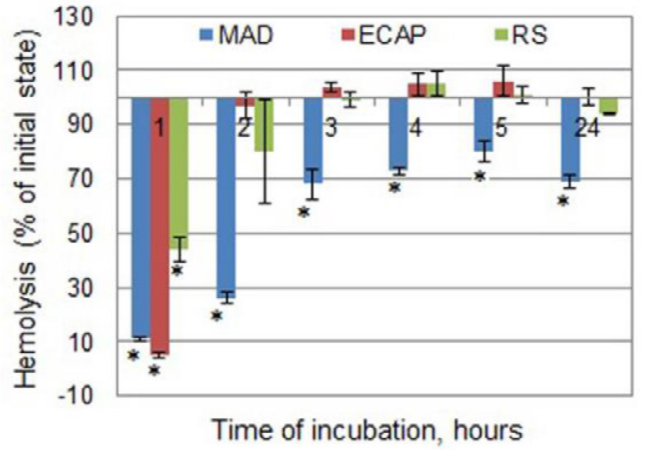

(a)

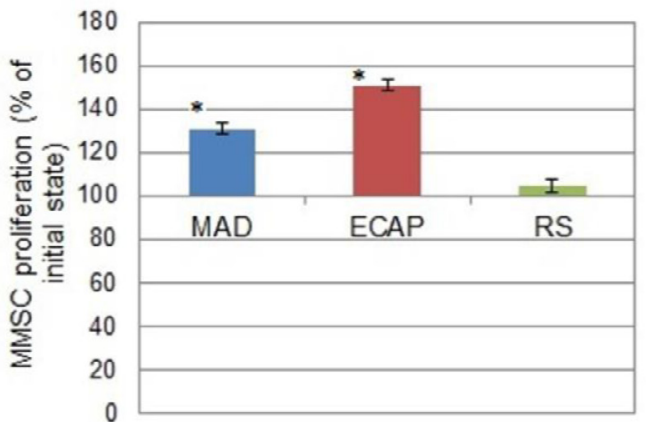

(c)

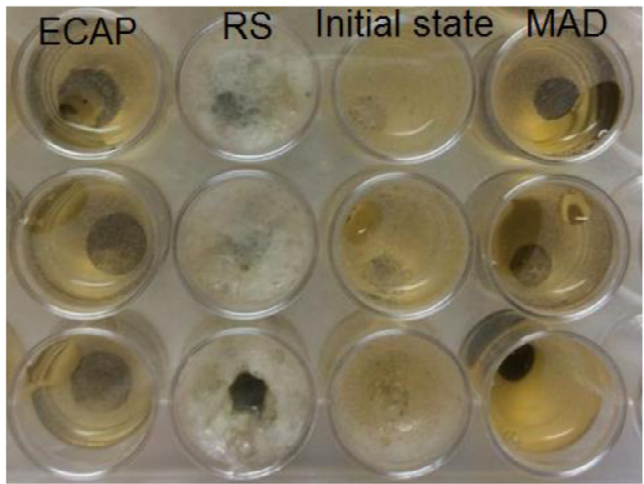

(e)

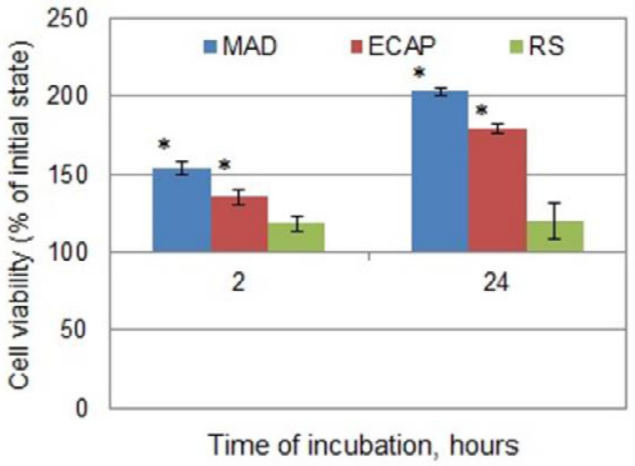

(b)

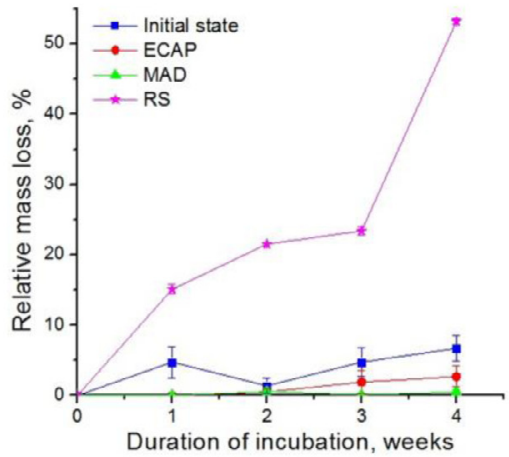

(d)

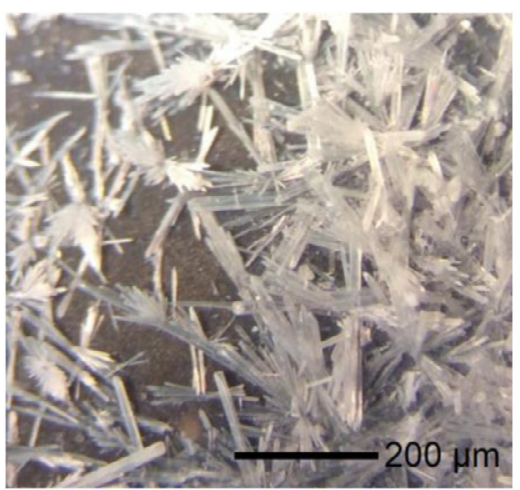

(f)

Fig. 5. The effect of the deformation processing of alloy WE43 on the biocompatibility in vitro (a) hemolysis, (b) WBCs viability, (c) MMSCs proliferation after 7 days of incubation, (d) mass loss of the alloy in $\mathrm{FBS}$ at $37^{\circ} \mathrm{C}$, (e) hydrogen gas evolution during the degradation process after 6 days of incubation, (f) sample surface after incubation in FBS (before removal of corrosion products). The hemolysis, WBCs viability and MMSCs proliferation values were calculated as a percentage of the respective values for the alloy in the initial state defined as the initial state for ECAP processing.

of cells with samples of the alloy. In addition, a study of the alloy degradation rate in fetal bovine serum at $37^{\circ} \mathrm{C}$ was conducted (Fig. 5).

The dynamics of hemolysis was evaluated after $1,2,3,4,5$, and 24 hours of alloy samples co-incubation with RBCs. Fig. 5a shows the change in the hemolytic activity of WE43 alloy in the initial state after deformation processing. After a short incubation period (one hour) all the deformation treatments samples of the alloy caused a significantly lower level of hemolysis compared to the alloy in the initial state. After two hours of co-incubation, significantly lower hemolysis was initiated by samples of the alloy processed by MAD and ECAP. With a further extension of the incubation time, a retardation of hemolysis was observed only for samples processed by MAD, while ECAP and RS had no significant effect on the activity of the alloy compared to the initial state.

Based on the analysis of changes in the MTT activity, it was found that WE43 treated by MAD and ECAP depressed the cell viability to a lesser extent than in the initial state. This improvement of cellular response to WE43 associated with deformation-induced grain refinement was more pronounced for a longer co-incubation time of $24 \mathrm{~h}$ than for a two-hour exposure. No significant change in cell viability as a result of co-incubation was observed for WE43 processed by RS.

A similar trend was established by studying the effect of the alloy processed by severe deformation on the MMSCs proliferation. After 7 


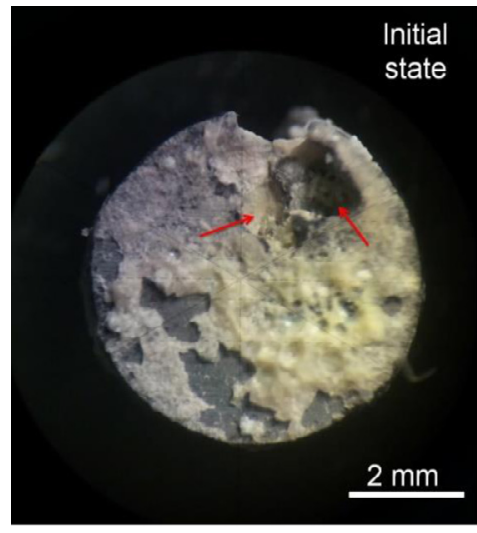

(a)

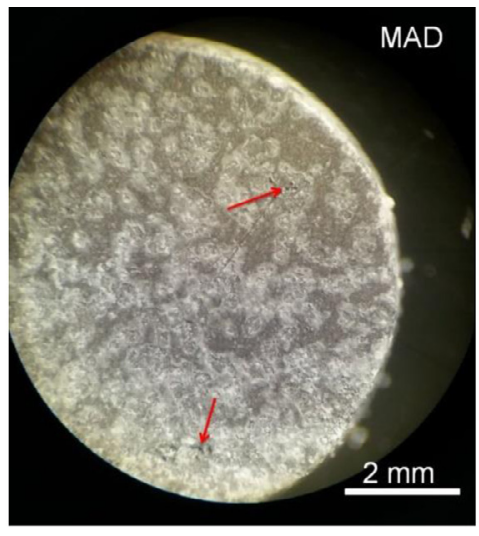

(b)

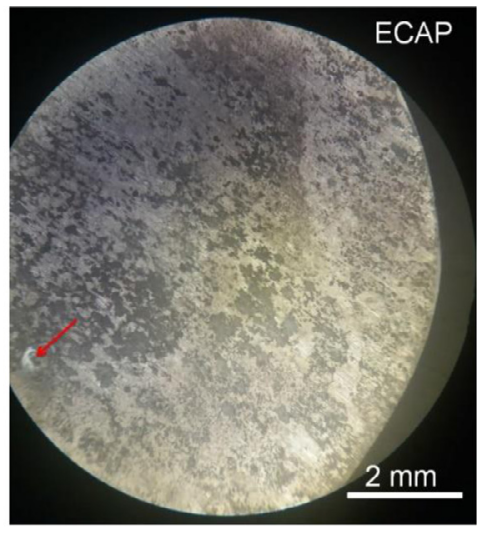

(c)

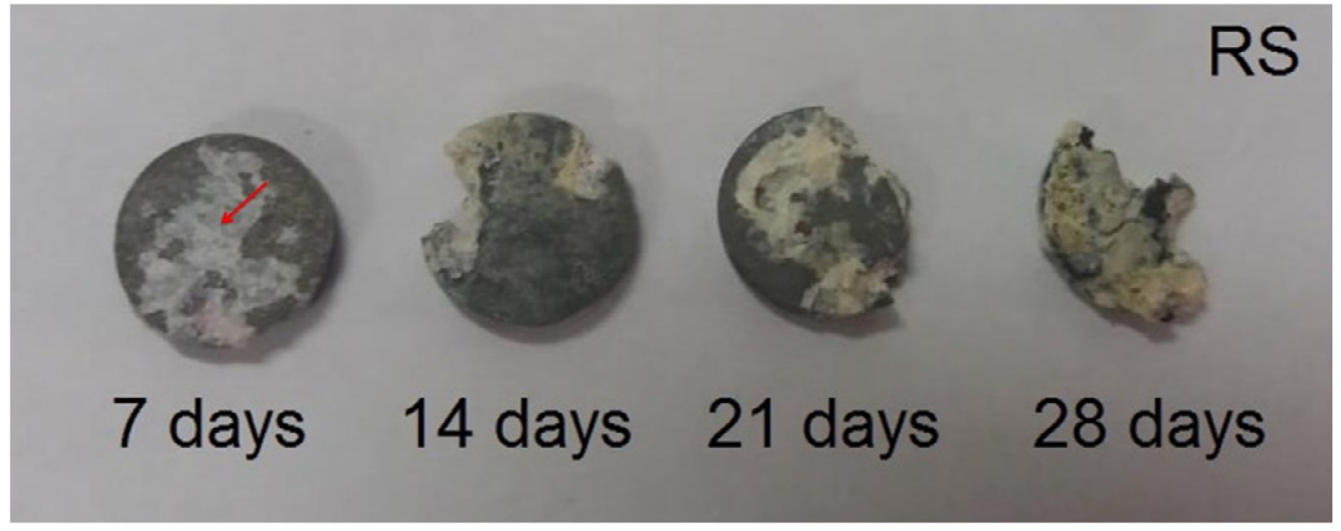

(d)

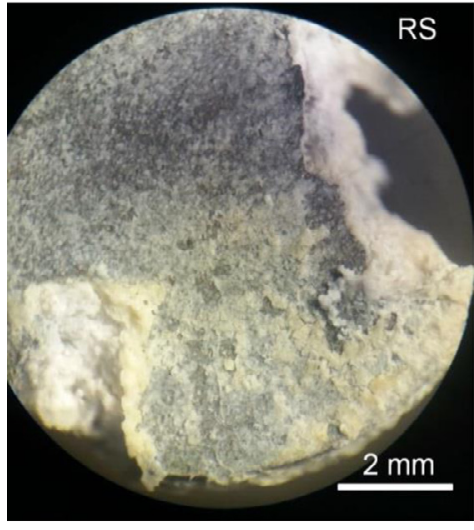

(e)

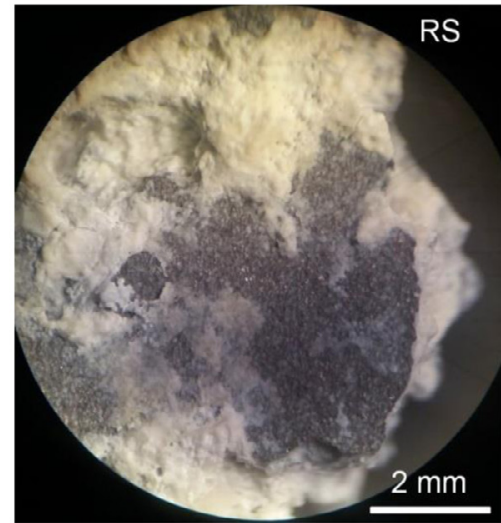

(f)

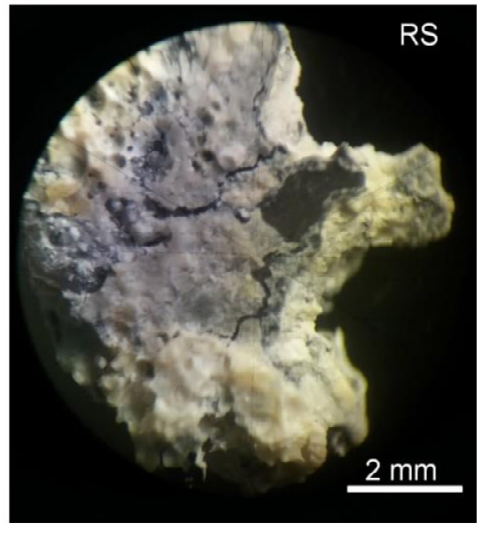

(g)

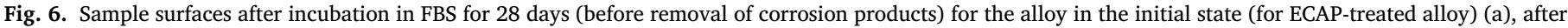

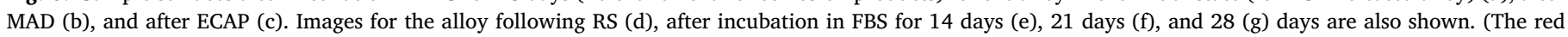
arrows indicate localization sites of corrosion).

days of incubation it was found that MMSCs proliferation on WE43 surface was boosted by processing by MAD and ECAP. By contrast, the RS treatment almost did not change this biocompatibility parameter.

Fig. $5 \mathrm{~d}$ shows the variation of the corrosion resistance with the incubation time for the various states of alloy WE43 in terms of the relative mass loss. The curves demonstrate that ECAP and MAD treatment hardly caused any change of the sample mass over the test duration of 28 days. After four weeks of incubation in FBS, the relative mass loss of the alloy after ECAP and MAD was $2.70 \pm 1.48 \%$ and $0.51 \pm 0.36 \%$, respectively - much less than the mass loss of the samples in the initial condition
$(6.71 \pm 1.85 \%)$. The effect of RS processing was significantly different, both qualitatively and quantitatively. A strong localization of corrosion with the formation of pitting wells was observed. Accordingly, the relative mass loss after 4 weeks of incubation in this condition was the largest and amounted to $53.22 \pm 0.44 \%$. In addition, prolonged evolution of hydrogen was observed during incubation in the test medium for the alloy in the initial state and after RS (Fig. 5e). Profuse gas formation can have negative consequences for biocompatibility of implants in vivo and is obviously undesirable. Inspection of the surface morphology of the MAD- and ECAP-treated samples showed that they did not exhibit 
any significant changes after 28 days of incubation in FBS. In general, the samples degraded practically homogeneously. Only weak signs of localization of corrosion were observed (Fig. 6 b, c). In contrast, pronounced pitting is clearly visible on the alloy samples in the initial state after 28 days of incubation in FBS (Fig. 6 a). Major degradation was concentrated at these surface locations. In the case of RS-treated alloy, localization of corrosion occurred already at the initial stage of incubation, and the degradation proceeded mainly from one edge of the sample with an increase in the incubation time (Fig. $6 \mathrm{~d}-\mathrm{g}$ ). It should also be noted that the surfaces of all samples were covered with an extremely heterogeneous membrane consisting mainly of the degradation products of the alloy.

\section{Discussion}

The results presented in the previous section show that all three deformation treatments studied (ECAP, MAD, and RS) lead to a significant strengthening of the WE43 alloy yielding UTS values that can be as high as $416 \mathrm{MPa}$. It should be noted that in the case of ECAP and MAD, in addition to an increase in the strength of the alloy (up to $300 \mathrm{MPa}$ ), an increase in tensile elongation to, respectively, 13.2 and $17.2 \%$, was observed. An increase in the strength of the alloy can be associated with the formation of a UFG structure as a result of severe plastic deformation. The best performing material, with the highest strength value (416 $\mathrm{MPa}$ ), was the alloy processed by RS. In this case, alongside the most dispersed microstructure with an average grain size of $0.61 \pm 0.19 \mu \mathrm{m}$, a large number of deformation twins were also observed. They contribute to the strengthening of the alloy but slightly decrease its tensile ductility (Table 2). However, this reduction of ductility is not dramatic, and is outweighed by the significant gain in strength. A similar strengthening effect was also reported for the WE43 alloy processed by high pressure torsion (HPT) [59]. Unfortunately, the UTS of $390 \mathrm{MPa}$ achieved by combining HPT with subsequent aging was accompanied with a drop of tensile ductility to almost zero. RS processing also caused a reduction of ductility, but the tensile elongation of more than $7 \%$ obtained by this kind of processing can still be regarded as satisfactory. The observed strengthening effects can be associated with grain refinement and, in the case of RS, with an additional contribution from deformation twinning. Although $\mathrm{Mg}_{41} \mathrm{Nd}_{5}$ intermetallic particles were formed in the alloy during deformation, their volume fraction was too small for their contribution to strengthening to be of significance.

An analysis of the texture induced by deformation processing was carried out to identify the causes of the observed increase in ductility. As shown earlier, a sharp prismatic texture was formed in alloy WE43 processed by ECAP. The calculation of the orientation factors of the slip systems and the main twinning system in hexagonal close-packed metals (an increase for basal and pyramidal slip and twinning and a decrease for prismatic slip) also shows that the probability of prismatic slip is increased, while that of basal and pyramidal slip and twinning weaken by ECAP (Table 3). Enhanced probability of dislocation slip on prismatic planes is known to be one of the main reasons for increased ductility of magnesium alloys.

As discussed above, after MAD processing the alloy did not have a pronounced texture. However, the calculation of the orientation factors showed that the probability of basal slip declined and that of prismatic slip rose as a result of processing. The overall outcome was an increase in the ductility of the alloy. An additional effect is an increase in the pyramidal slip and twinning. The latter may be associated with the presence of compressive stresses in the MAD process. However, unexpectedly, no deformation twins were detected in the microstructure after MAD. It is likely that the critical shear stresses in this case were so high that even a significant decrease in the orientation factor was insufficient to initiate the twinning process.

As already mentioned, high mechanical strength and sufficient corrosion resistance are an important prerequisite for the use of $\mathrm{Mg}$ alloys in medical implants. From this viewpoint, deformation processing producing an UFG structure is promising, as it leads to improved strength characteristics combined with adequate corrosion resistance. Indeed, the present study demonstrated the formation of a UFG structure and texture that gave rise to an increase in the strength of alloy WE43 without a loss of its corrosion resistance. As a matter of fact, in the case of ECAP and MAD the chemical corrosion rate was reduced and tensile ductility was increased, cf. Fig. 5 and Table 2.

Currently, the mechanisms underlying the observed corrosion resistance behavior cannot be considered as established. On the one hand, a decrease in the average grain size leads to an increase in the length of the grain boundaries exposed to the cell culture, which are essentially a defect in the crystal structure of the alloy whose increase should affect the corrosion resistance negatively through acceleration of the corrosion process. On the other hand, a high corrosion rate at an early stage of the process can accelerate the formation of a protective layer consisting of magnesium oxides and hydroxides, and subsequently slow down the corrosion process. In addition, the UFG structure with a high density of grain boundaries can become a barrier to the spread of pitting corrosion, which is also a serious problem with magnesium alloys [39]. Suppression of pitting will lead to a decrease in the localization of corrosion, which will reduce non-uniformity of the mechanical properties of an implant and hence the risk of failure during operation or premature fracture in service. There are several other factors whose interplay complicates the interpretation of the corrosion behavior. Among them is the occurrence of precipitation during the deformation processing. The structure of the alloy in the initial state consists of large grains of a supersaturated magnesium solid solution. In addition to grain refinement, the deformation process leads to the precipitation of dispersed intermetallic $\mathrm{Mg}_{41} \mathrm{Nd}_{5}$ particles, thus creating closely spaced micro galvanic couples with the Mg matrix, which enhances corrosion.

A factor that can influence corrosion resistance is surface roughness after the formation of an UFG structure [60], and therefore the surface roughness was (Table 4). The parameters measured included the average roughness (Ra), the root mean square roughness ( $\mathrm{Rq}$ ), the average maximum peak-to-valley value (Rz), the average maximum height of the profile (Rt), the kurtosis (Rku), and the skewness (Rsk).

As seen from Table 4, all three processing techniques employed, MAD, ECAP, and RS, brought about a significant decrease in the parameters Ra, Rt, Rz, and Rq. It is believed that the surface features defining the roughness profile are associated with differences in the bulk microstructure - in the condition of testing - in particular the reduced grain size and increased dislocation density. We note that the magnitude of the parameters Rku and Rsk is the greatest for the samples of WE43 processed by RS. This was probably the reason for the extremely high biodegradation rate in FBS for the extended incubation time of four weeks, and also the strong localization of corrosion. This behavior

Table 3

Orientation factors for alloy WE43 in the initial state and after processing.

\begin{tabular}{lllll}
\hline State of the alloy & Basal $\{0001\}<1120>$ & Prismatic $\{1010\}<1120>$ & Pyramidal $<\mathrm{c}+\mathrm{a}>$ & Twinning $\{10 \overline{1} 2\}\langle 10 \overline{1} \overline{1}\rangle$ \\
\hline Initial state for ECAP & 4.6 & 6.2 & 4.1 & 4.0 \\
ECAP & 6.0 & 4.3 & 5.0 & 5.0 \\
Initial state for MAD & 4.8 & 5.5 & 5.6 & 6.0 \\
MAD & 5.3 & 4.4 & 4.7 & 4.6 \\
\hline
\end{tabular}


Table 4

The surface roughness parameters for the WE43 alloy with various processing histories (The initial state for ECAP-treated alloy was used as a reference.).

\begin{tabular}{lllllll}
\hline State of the alloy & $\mathrm{Ra}, \mathrm{nm}$ & $\mathrm{Rt}, \mathrm{nm}$ & $\mathrm{Rz}, \mathrm{nm}$ & $\mathrm{Rq}, \mathrm{nm}$ & $\mathrm{Rku}$ & $\mathrm{Rsk}$ \\
\hline Initial state + Polishing & 356 & 3370 & 2540 & 434 & 3.03 & -0.69 \\
MAD + Polishing & 136 & 1055 & 1020 & 168 & 2.93 & -0.48 \\
ECAP + Polishing & 26 & 620 & 358 & 33 & 5.12 & -0.69 \\
RS + Polishing & 69 & 1950 & 1400 & 98 & 14.46 & -1.96 \\
\hline
\end{tabular}

is likely connected with the formation of deformation twins in WE43 deformed by RS. However, further research is needed to confirm this hypothesis.

Surface roughness also had an effect on the biocompatibility parameters. Thus, the observed decrease in hemolysis and increase in the cell viability of the alloy in the initial condition induced by deformation processing is correlated with a decrease in the values of Ra, Rt, Rz, and Rq. However, these phenomena may also be associated with a decrease in the degradation rate of the alloy after the deformation treatments in the early stages of incubation. Since a decrease in the degradation rate led to a reduction of the rate of hydrogen evolution and less alkalization of the culture medium, biocompatibility might have been improved. The likelihood of this effect of the degradation rate on biocompatibility is also confirmed by the results of the assessment of changes in MMSC proliferation after 7 days of incubation with the studied alloy. In our experiments, a significant increase in the degradation rate was observed for the initial and swaged alloy after 7 days of incubation, which was accompanied with profuse hydrogen evolution (Fig. 5e). By contrast, the degradation rate of the alloy processed by MAD and ECAP hardly changed. The results on the MMSC proliferation dynamic, in general, correlate with these data. It was proven that MAD- and ECAP-treated WE43 promoted the proliferation in vitro of these cells compared to the alloy in the initial state. The behavior of the RS processed alloy tells us a different story. There are reasons to believe that the acceleration of the biodegradation process for this condition inhibits cell proliferation due to an increase in the $\mathrm{pH}$ of the medium. The ensuing deterioration of the properties of the growth culture medium causes mechanical repulsion of cells from the surface of the alloy samples due to the rapid formation of numerous hydrogen bubbles. In addition, corrosion of the alloy was also accompanied with the formation of a surface coating with needle-like crystals of magnesium hydroxide $\mathrm{Mg}(\mathrm{OH})_{2}$ (Fig. 5f). Possibly this inhibited the proliferation of MMSCs by mechanically damaging the cells when they adhered to the surface.

Summarizing the data obtained, we can conclude that the structure refinement to a UFG state by the severe plastic deformation techniques considered enables a significant improvement of the properties of alloy WE43, albeit to different degrees. Along with an increase in strength due to a pronounced grain refinement and the formation of deformation twins in the case of rotary swaging processed material, the ductility of the alloy can also be increased in the presence of a favorable texture. Furthermore, changes in the microstructure by severe plastic deformation techniques, using the right processing regime, can also lead to improved corrosion resistance, which in turn improves biocompatibility in vitro. That is to say, the formation of the UFG structure may not only enhance the strength characteristics of alloy WE43, but also improve its in-service properties, significantly expanding the possibilities of using the alloy in medical implants. It should be noted that the formation of deformation twins by rotary swaging, while producing the best results in terms of strength, accelerates corrosion. However, it may be possible to solve this problem by designing new temperature and deformation regimes. In any case, investigations aiming at further improvement of the property profile of alloy WE43 relevant for implant applications are believed to be the order of the day. Further in vitro experiments need to be complemented with in vivo assays, and we are planning such studies for future work.

\section{Conclusions}

1 It was revealed that mechanical processing of magnesium alloy WE43 by various methods of severe plastic deformation leads to the formation of an ultrafine-grained structure. Severe plastic deformation resulted in an average grain size in the range of $\sim 0.6-1 \mu \mathrm{m}$, and $\mathrm{Mg}_{41} \mathrm{Nd}_{5}$ phase intermetallic particles with an average size of $0.30-0.45 \mu \mathrm{m}$.

2 Through processing by rotary swaging, it was possible to increase the UTS of the alloy to $416 \mathrm{MPa}$ with a small decrease in ductility to $\sim 7.9 \%$. In the case of ECAP and MAD processing, grain refinement led to an increase in tensile ductility to about $\sim 17.2 \%$ due to the activation of prismatic slip, along with a concomitant increase in the UTS to $\sim 300 \mathrm{MPa}$.

3 Grain refinement caused by all three deformation methods employed did not result in a deterioration of the resistance of WE43 to electrochemical corrosion. In fact, results revealed that ECAP and MAD led to a decrease of corrosion rate in both short-term and four-week long experiments. However conversely, processing by rotary swaging was found to accelerate biocorrosion of WE43 in a simulated body fluid (fetal bovine serum).

4 It was shown that grain refinement by ECAP and, especially, MAD improved the biocompatibility of the initial alloy, impeding hemolysis, WBCs viability, and MMSC proliferation. The deformation treatment of WE43 in the initial state by RS contributed to a short-term decrease in its hemolytic activity.

5 Overall, the present study indicated that the properties of WE43, an alloy that has already been deemed as a candidate relevant to medical implants, can be improved significantly, making the magnesium alloy WE43 particularly significant in terms of a leading property profile.

\section{Declaration of Competing Interest}

The authors declare no conflict of interest.

\section{Acknowledgments}

Funding support of investigations of microstructure, mechanical performance, corrosion resistance and biocompatibility was provided by the Russian Science Foundation (project \#18-45-06010). Part of this work relating to studies of texture was carried out within the governmental task \#075-00947-20-00.

\section{References}

[1] M.B. Kannan, R.K. Raman, In vitro degradation and mechanical integrity of calcium containing magnesium alloy in modified simulated body fluid, Biomaterials. 29(2008) 2306-2314. https://doi.org/10.1016/j.biomaterials.2008.02.003.

[2] X.J. Wang, Y.C. Li, P.D. Hodgson, C. Wen, Nano- and macro-scale characterization of the mechanical properties of bovine bone, Mater. Forum. 31(2007) 156-159. http://hdl.handle.net/10536/DRO/DU:30007337.

[3] W. Ding, Opportunities and challenges for the biodegradable magnesium alloys as next-generation biomaterials, Regen. Biomater 3 (2) (2016) 79-76, doi:10.1093/rb/rbw003.

[4] S. Virtanen, Biodegradable $\mathrm{Mg}$ and $\mathrm{Mg}$ alloys: Corrosion and biocompatibility, Mater. Sci. Eng. B. 176(2011) 1600-1608. https://doi.org/10.1016/j.mseb.2011.05.028. 
[5] M. Niinomi, M. Nakai, J. Hieda, Development of new metallic alloys for biomedical applications, Acta Biomat. 8(2012) 3888-3903. https://doi.org/10.1016/j.actbio.2012.06.037.

[6] Y.F. Zheng, X.N. Gu, F. Witte, Biodegradable metals, Mater. Sci. Eng.R. 77(2014) 1-34. https://doi.org/10.1016/j.mser.2014.01.001.

[7] X. Li, X. Liu, S. Wu, K.W.K. Yeung, Y. Zheng, P.K. Chu, Design of magnesium alloys with controllable degradation for biomedical implants: From bulk to surface, Acta Biomater. 45(2016) 2-30. https://doi.org/10.1016/j.actbio.2016.09.005.

[8] A. Atrens, M. Liu, N.I.Z. Abidin, Corrosion mechanism applicable to biodegradable magnesium implants, Mater. Sci. Eng. B. 176(2011) 1609-1636. https://doi.org/10.1016/j.mseb.2010.12.017.

[9] Y. Chen, Z. Xu, C. Smith, J. Sankar, Recent advances on the development of magnesium alloys for biodegradable implants, Acta Biomater 10 (2014) 4561-4573, doi:10.1016/j.actbio.2014.07.005.

[10] M.S. Uddin, C. Hall, P. Murphy, Surface treatments for controlling corrosion rate of biodegradable $\mathrm{Mg}$ and Mg-based alloy implants, Sci. Technol. Adv. Mat 16 (5) (2015) 053501, doi:10.1088/1468-6996/16/5/053501.

[11] A. Atrens, G.-L. Song, M. Liu, Z. Shi, F. Cao, M.S. Dargusch, Review of recent developments in the field of magnesium corrosion, Adv. Eng. Mater. 17(2015) 400-453. https://doi.org/10.1002/adem.201400434.

[12] J. Chen, L.Tan, X. Yu, I.P. Etim, M. Ibrahim, K. Yang, Mechanical properties of magnesium alloys for medical application: A review, J. Mech. Behav. Biomed. 87(2018) 68-79. https://doi.org/10.1016/j.jmbbm.2018.07.022.

[13] S.J. Meng, H. Yu, S.D. Fan, Y.M. Kim, S.H. Park, W.M. Zhao, B.S. You, K.S. Shin, A high-ductility extruded Mg-Bi-Ca alloy, Mater. Lett. 261 (2020) 127066, doi:10.1016/j.matlet.2019.127066.

[14] Y. Song, D. Shan, E.-H. Han, Pitting corrosion of a Rare Earth Mg alloy GW93, J. Mater. Sci. Technol. 33 (9) (2017) 954-960, doi:10.1016/j.jmst.2017.01.014.

[15] H.J. Martin, M.F. Horstemeyer, P.T. Wang, Structure-property quantification of corrosion pitting under immersion and salt-spray environments on an extruded AZ61 magnesium alloy, Corros. Sci. 53 (4) (2011) 1348-1361, doi:10.1016/j.corsci.2010.12.025.

[16] M.P. Staiger, A.M. Pietak, J. Huadmai, G. Dias, Magnesium and its alloys as orthopedic biomaterials: a review, Biomaterials 27 (2006) 1728-1734, doi:10.1016/j.biomaterials.2005.10.003.

[17] F. Witte, The history of biodegradable magnesium implants: a review, Acta Biomater 6 (2010) 1680-1692, doi:10.1016/j.actbio.2010.02.028.

[18] F. Witte, V. Kaese, H. Haferkamp, E. Switzer, A. Meyer-Lindenberg, C.J. Wirth, H. Windhagen, In vivo corrosion of four magnesium alloys and the associated bone response, Biomaterials 26 (2005) 3557-3563, doi:10.1016/j.biomaterials.2004.09.049.

[19] C. Op't Hoog, N. Birbilis, Y. Estrin, Corrosion of pure $\mathrm{Mg}$ as a function of grain size and processing route, Adv. Eng. Mater 10 (2008) 579-582, doi:10.1002/adem.200800046.

[20] C. Op't Hoog, N. Birbilis, M.X. Zhang, Y. Estrin, Surface grain size effects on the corrosion of magnesium, Key Eng. Mater 384 (2008) 229-240, doi:10.4028/www.scientific.net/KEM.384.229.

[21] Z. Liu, R. Schade, B. Luthringer, N. Hort, H. Rothe, S. Müller, K. Liefeith, R. Willumeit-Römer, F. Feyerabend, Influence of the microstructure and silver content on degradation, cytocompatibility, and antibacterial properties of Magnesium-Silver alloys in vitro, Oxid. Med. Cell Longev (2017) 8091265, doi: $10.1155 / 2017 / 8091265$

[22] A.F. Cipriano, A. Sallee, M. Tayoba, M.C.Cortez Alcaraz, A. Lin, R.-G. Guan, Z.-Y. Zhao, H. Liu, Cytocompatibility and early inflammatory response of human endothelial cells in direct culture with Mg-Zn-Sr alloys, Acta Biomater. 48(2017) 499-520. https://doi.org/10.1016/j.actbio.2016.10.020.

[23] X.N. Gu, N. Li, Y.F. Zheng, L. Ruan, In vitro degradation performance and biological response of a Mg-Zn-Zr alloy, Mater. Sci. Eng. B 176 (20) (2011) 1778-1784, doi:10.1016/j.mseb.2011.05.032.

[24] Y. Pan, S. He, D. Wang, D. Huang, T. Zheng, S. Wang, P. Dong, C. Chen, In vitro degradation and electrochemical corrosion evaluations of microarc oxidized pure $\mathrm{Mg}, \mathrm{Mg}-\mathrm{Ca}$ and $\mathrm{Mg}-\mathrm{Ca}-\mathrm{Zn}$ alloys for biomedical applications, Mater. Sci. Eng. C 47 (2015) 85-96, doi:10.1016/j.msec.2014.11.048.

[25] Z. Li, X. Gu, S. Lou, Y. Zheng, The development of binary Mg-Ca alloys for use as biodegradable materials within bone, Biomaterials 29 (10) (2008) 1329-1344, doi:10.1016/j.biomaterials.2007.12.021.

[26] N.A. Agha, Liu Z, F. Feyerabend, R. Willumeit-Römer, B. Gasharova, S. Heidrich, B. Mihailova, The effect of osteoblasts on the surface oxidation processes of biodegradable Mg and Mg-Ag alloys studied by synchrotron IR microspectroscopy, Mater. Sci. Eng. C. 91 (2018) 659-668, doi:10.1016/j.msec.2018.06.001.

[27] A. Myrissa, N.A. Agha, Y. Lu, E. Martinelli, J. Eichler, G. Szakács, C. Kleinhans, R. Willumeit-Römer, U. Schäfer, A.-M. Weinberg, In vitro and in vivo comparison of binary Mg alloys and pure Mg, Mater. Sci. Eng.C 61 (2016) 865-874, doi:10.1016/j.msec.2015.12.064.

[28] A. Witecka, A. Boguck, A. Yamamoto, K. Máthis, T. Krajňák, J. Jaroszewicz, W. Święszkowski, In vitro degradation of ZM21 magnesium alloy in simulated body fluids, Mater. Sci. Eng. C 65 (2016) 59-69, doi:10.1016/j.msec.2016.04.019.

[29] L. Xu, G. Yu, E. Zhang, F. Pan, K. Yang, In vivo corrosion behavior of Mg-Mn-Zn alloy for bone implant application, J. Biomed. Mater. Res 83A (3) (2007) 703-711, doi:10.1002/jbm.a.31273.

[30] Y. Li, C. Wen, D. Mushahary, R. Sravanthi, N. Harishankar, G. Pande, P. Hodgson, $\mathrm{Mg}-\mathrm{Zr}-\mathrm{Sr}$ alloys as biodegradable implant materials, Acta Biomater 8 (8) (2012) 3177-3188, doi:10.1016/j.actbio.2012.04.028.

[31] S.S. Nene, B.P. Kashyap, N. Prabhu, Y. Estrin, T. Al-Samman, Biocorrosion and biodegradation behavior of ultralight $\mathrm{Mg}-4 \mathrm{Li}-1 \mathrm{Ca}$ (LC41) alloy in simulated body fluid for degradable implant applications, J. Mater. Sci. 50 (8) (2015) 3041-3050, doi:10.1007/s10853-015-8846-y.

[32] Y. Liu, Y. Wu, D. Bian, S. Gao, S. Leeflang, H. Guo, Y. Zheng, J. Zhou, Study on the $\mathrm{Mg}$-Li-Zn ternary alloy system with improved mechanical properties, good degradation performance and different responses to cells, Acta Biomater 62 (2017) 418-433, doi:10.1016/j.actbio.2017.08.021.

[33] F. Witte, V. Kaese, H. Haferkamp, E. Switzer, A. Meyer-Lindenberg, C.J. Wirth, H. Windhagen, In vivo corrosion of four magnesium alloys and the associated bone response, Biomaterials 26 (2005) 3557-3563, doi:10.1016/j.biomaterials.2004.09.049.

[34] R. Waksman, R. Pakala, P.K. Kuchulakanti, R. Baffour, D. Hellinga, R. Seabron, F.O. Tio, E. Wittchow, S. Hartwig, C. Harder, R. Rohde, B. Heublein, A. Andreae, K.H. Waldmann, A. Haverich, Safety and efficacy of bioabsorbable magnesium alloy stents in porcine coronary arteries, Catheter. Cardiovasc. Interv 68 (2006) 607-617, doi:10.1002/ccd.20727.

[35] I. Marco, A. Myrissa, E. Martinelli, F. Feyerabend, R. Willumeit-Römer, A.M. Weinberg, O. Van der Biest, In vivo and in vitro degradation comparison of pure $\mathrm{Mg}$, Mg-10Gd and Mg-2Ag: a short term study, Eur. Cell Mater. 33(2017) 90-104. doi: 10.22203/eCM.v033a07.

[36] C.L. Liu, Y.C. Xin, G.Y. Tang, P.K. Chu, Influence of heat treatment on degradation behavior of bio-degradable die-cast AZ63 magnesium alloy in simulated body fluid, Mater. Sci. Eng. A 456 (2007) 350-357, doi:10.1016/j.msea.2006.12.020.

[37] F. Witte, J. Fischer, J. Nellesen, H.-A. Crostack, V. Kaese, A. Pisch, F. Beckmann, $\mathrm{H}$. Windhagen, In vitro and in vivo corrosion measurements of magnesium alloys, Biomaterials 27 (2006) 1013-1018, doi:10.1016/j.biomaterials.2005.07.037.

[38] J. Walker, S. Shadanbaz, T.B.F. Woodfield, M.P. Staiger, G.J. Dias., Magnesium biomaterials for orthopedic application: A review from a biological perspective, J. Biomed. Mater. Res. B. 102 (2014) 1316-1331, doi:10.1002/jbm.b.33113.

[39] D. Ahmadkhaniha, M. Fedel, M. Heydarzadeh Sohi, F. Deflorian, Corrosion behavior of severely plastic deformed magnesium based alloys: a review, Surf. Eng. Appl. Elect 53 (5) (2017) 439-448, doi:10.3103/S1068375517050039.

[40] R.Z. Valiev, A.P. Zhilyaev, T.G. Langdon, Bulk Nanostructured Materials: Fundamentals and Applications, Wiley, Hoboken, 2014.

[41] R.Z. Valiev, R.K. Islamgaliev, I.V. Alexandrov, Bulk nanostructured materials from severe plastic deformation, Prog. Mater. Sci 45 (2000) 103-189 https://doi.org/10.1016/S0079-6425(99)00007-9.

[42] Yu.S. Radyuchenko, Rotary Swaging. Processing of Parts with Rotary Swaging Machines, Mashgiz, Moscow, 1962 in Russian.

[43] W.M. Gan, Y.D. Huang, R. Wang, G.F. Wang, A. Srinivasan, H.-G. Brokmeier, N. Schell, K.U. Kainer, N. Hort, Microstructures and mechanical properties of pure $\mathrm{Mg}$ processed by rotary swaging, Mater. Des. 63(2014) 83-88. https://doi.org/10.1016/j.matdes.2014.05.057.

[44] W.M. Gan, Y.D. Huang, R. Wang, Z.Y. Zhong, N. Hort, K.U. Kainer, N. Schell, H.-G. Brokmeier, A. Schreyer, Bulk and local textures of pure magnesium processed by rotary swaging, J. Magnes. Alloys. 1 (2013) 341-345, doi:10.1016/j.jma.2013.12.004.

[45] S.V. Zherebtsov, G.A. Salishchev, R.M. Galeyev, O.R. Valiakhmetov, S. Yu, S.L. Mironov, Semiatin, Production of submicrocrystalline structure in large-scale Ti-6Al-4V billet by warm severe deformation processing, Scr. Mater. 5 (12) (2004) 1147-1151, doi:10.1016/j.scriptamat.2004.08.018.

[46] A. Salandari-Rabori, A. Zarei-Hanzaki, S.M. Fatemi, M. Ghambari, M. Moghaddam, Microstructure and superior mechanical properties of a multi-axially forged WE magnesium alloy, J. Alloys Compd 693 (2017) 406-413, doi:10.1016/j.jallcom.2016.09.198.

[47] M. Diez, H.-E. Kim, V. Serebryany, S. Dobatkin, Y. Estrin, Improving the mechanical properties of pure magnesium by three-roll planetary milling, Mater. Sci. Eng. A 612 (2014) 287-292, doi:10.1016/j.msea.2014.06.061.

[48] X.N. Gu, W.R. Zhou, Y.F. Zheng, Y. Cheng, S.C. Wei, S.P. Zhong, T.F. Xi, L.J. Chen, Corrosion fatigue behaviors of two biomedical Mg alloys-AZ91D and WE43-In simulated body fluid, Acta Biomater 6 (12) (2010) 4605-4613, doi:10.1016/j.actbio.2010.07.026.

[49] S.V. Dobatkin, E.A. Lukyanova, N.S. Martynenko, N. Yu, M.V Anisimova, M.V. Kiselevskiy, N.Yu Gorshenkov, G.I.Raab Yurchenko, V.S. Yusupov, N. Birbilis, G.A. Salishchev, Y.Z. Estrin, Strength, corrosion resistance, and biocompatibility of ultrafinegrained $\mathrm{Mg}$ alloys after different modes of severe plastic deformation, IOP Conf. Series: Mater. Sci. 194 (2017) 012004, doi:10.1088/1757-899X/194/1/012004.

[50] N.S. Martynenko, E.A. Lukyanova, V.N. Serebryany, M.V. Gorshenkov, I.V. Shchetinin, G.I. Raab, S.V. Dobatkin, Y. Estrin, Increasing strength and ductility of magnesium alloy WE43 by equal-channel angular pressing, Mater. Sci. Eng 712 (2018) 625-629, doi:10.1016/j.msea.2017.12.026.

[51] N.S. Martynenko, E.A. Luk'yanova, M.M. Morozov, V.S. Yusupov, S.V. Dobatkin, Yu.Z. Estrin, A Study of the structure, mechanical properties and corrosion resistance of magnesium alloy WE43 after rotary swaging, Met. Sci. Heat Treat 60 (3-4) (2018) 253-258, doi:10.1007/s11041-018-0269-3.

[52] E.A. Lukyanova, N.S. Martynenko, V.N. Serebryany, A.N. Belyakov, L.L. Rokhlin, S.V. Dobatkin, Yu.Z. Estrin, Structure and Mechanical and Corrosion Properties of a Magnesium Mg-Y-Nd-Zr Alloy after High Pressure Torsion, Russ.Metall. (Metally) 2017 (11) (2017) 912-921, doi:10.1134/S0036029517110088.

[53] T.I. Savyolova, S.F. Kourtasov, ODF restoration by orientation grid, Mater. Sci. Forum (2005) 459-457, 301-306. DOI 10.4028/www.scientific.net/MSF.495-497.301.

[54] V.N. Serebryany, L.L. Rokhlin, A.N. Monina, Texture and anisotropy of mechanical properties of the magnesium alloy of Mg-Y-Gd-Zr system, Inorg. Mater. Appl. Res 5 (2) (2014) 116-123, doi:10.1134/S207511331402018X. 
[55] E. Lukyanova, N. Anisimova, N. Martynenko, M. Kiselevsky, S. Dobatkin, Y. Estrin, Features of in vitro and in vivo behaviour of magnesium alloy WE43, Mater. Lett 215 (2018) 308-311 doi.org/, doi:10.1016/j.matlet.2017.12.125.

[56] F.S. Senatov, M.V. Gorshenkov, S.D. Kaloshkin, V.V. Tcherdyntsev, N.Yu. Anisimov, A.N. Kopylov, M.V. Kiselevsky, Biocompatible polymer composites based on ultrahigh molecular weight polyethylene perspective for cartilage defects replacement, J. Alloy. Compd. 586 (SUPPL. 1) (2014) 544-547 doi.org/, doi:10.1016/j.jallcom.2012.10.014.

[57] J.N. Yaochite, K.W. De Lima, C. Caliari-Oliveira, P.V. Bonini Palma, C.E. Barra Couri, B.P. Simões, D.T. Covas, J.C. Voltarelli, M.C. Oliveira, E.A. Donadi, K.C. Ribeiro Malmegrim, Multipotent mesenchymal stromal cells from patients with newly diagnosed type 1 diabetes mellitus exhibit preserved in vitro and in vivo immunomodulatory properties, Stem. Cell Research.Therapy 7 (1) (2016) 7-14 doi.org/, doi:10.1186/s13287-015-0261-4.
[58] J. Groebner, Magnesium-Neodymium-Yttrium, in: G. Effenberg, F. Aldinger, P. Rog] (Eds.), A Comprehensive Compendium of Evaluated Constitutional Data and Phase Diagrams, 18, Stuttgart, 2001, pp. 324-335.

[59] H. Okamoto, Mg-Nd, J. Phase Equil, Diffusion 28 (4) (2007) 405, doi:10.1007/s11669-013-0233-2.

[60] A.E. Medvedev, A. Neumann, H.P. Ng, R. Lapovok, C. Kasper, T.C. Lowe, V.N. Anumalasetty, Y. Estrin, Combined effect of grain refinement and surface modification of pure titanium on the attachment of mesenchymal stem cells and osteoblast-like SaOS-2 cells, Mater. Sci. Eng.C 71 (2017) 746-757, doi:10.1016/j.msec.2016.10.035. 